\title{
Mutations in Pea seedborne mosaic virus Genome-Linked Protein VPg Alter Pathotype-Specific Virulence in Pisum sativum
}

\author{
Britta Borgstrøm and I. Elisabeth Johansen \\ Biotechnology Group, Danish Institute of Agricultural Sciences, Thorvaldsensvej 40, 1., DK-1871 \\ Frederiksberg C, Denmark \\ Submitted 15 November 2000; Accepted 12 February 2001.
}

\begin{abstract}
Pisum sativum plant introduction (PI) line 269818 is resistant to potyvirus pea seedborne mosaic virus (PSbMV) isolates, categorized as pathotype $P 1$, and is susceptible to pathotype $\mathbf{P 4}$ isolates. This difference in infectivity is determined by the viral genome-linked protein (VPg) cistron. Mutational analysis of VPg of PSbMV isolates DPD1 and NY representing pathotypes $P 1$ and $P 4$ revealed that codon changes affecting amino acids 105 to 117 in the central region of VPg influenced virulence on PI 269818. In contrast, infectivity on pea cultivar Dark Skinned Perfection, which is susceptible to both pathotypes, was not affected by the mutations. Mutants overcoming resistance in PI 269818 were analyzed for changes in the VPg coding region upon passage through PI 269818 and Dark Skinned Perfection. Adaptive changes were observed only upon passage through PI 269818 and only at codons from amino acid 105 to 117. Expression of DPD1 VPg in PI 269818 did not affect infection by NY, which suggests that VPg from DPD1 is not an elicitor of a general resistance response. The results are compatible with the hypothesis that viral amplification depends upon the interaction between VPg and a host factor.
\end{abstract}

Additional keywords: recessive resistance, replication, sbm-1.

Plants are subject to pathogen attacks from various microorganisms such as fungi, bacteria, and viruses. Consequently, natural defense mechanisms evolved. Viruses are dependent completely upon the intracellular environment of host cells, and the host provides factors that support virus amplification and spread. Among viruses, the genus Potyvirus is the largest, accounting for approximately $30 \%$ of known viral plant pathogens (Riechmann et al. 1992). Recessive resistance genes appear to occur more frequently to potyviruses than to other plant virus genera (Revers et al. 1999). Recessive resistance is likely a result of passive mechanisms resulting from the absence or modification of a host factor required for infection (Fraser 1992). This assumption recently has been supported by the demonstration that recessive $v a$ alleles conferring resistance to several potyviruses are associated with large

Corresponding author: E. Johansen; E-mail: e.johansen@dias.kvl.dk deletions at the Va locus in tobacco (Noguchi et al. 1999). Alternatively, the host factor may be a negative regulator of resistance, as suggested for the Mlo gene product in barley, where mlo homozygosity is associated with nonrace-specific resistance to the powdery mildew fungus Erysiphe graminis f. sp. hordei (Freialdenhoven et al. 1996).

To establish infection, a potyvirus needs to complete three crucial steps inside the plant: genome amplification, cell-tocell movement, and long-distance transport (Revers et al. 1999). All three steps are affected by recessive resistance mechanisms. Replication of the tobacco etch virus (TEV) isolate TEV-HAT is abolished in the pepper Capsicum annuиm cv. Dempsey homozygous for the resistance gene $e t^{\mathrm{a}}$ (Deom et al. 1997). In Capsicum chinense, the resistance gene pvrl interferes with replication of Pepper mottle virus (PepMoV) and TEV-HAT (Murphy et al. 1998). In the Pisum sativum line PI 269818 homozygous for the pathotype-specific resistance gene $s b m-1$, replication of pea seedborne mosaic virus (PSbMV) pathotype P1 could not be detected in inoculated leaves, suggesting that $s b m-1$ resistance operates at the single-cell level (Keller et al. 1998). Cell-to-cell movement of Tobacco vein mottling virus (TVMV) and Potato virus $Y$ (PVY) is blocked in Nicotiana tabacum cultivars TN86 and VAM (Gibb et al. 1989; Masuta et al. 1999). Both tobacco cultivars are homozygous for the resistance allele $v a$, and the resistance is associated with a replication reduction (Gibb et al. 1989; Masuta et al. 1999). Cell-to-cell movement of a pepper isolate of PVY pathotype $\mathrm{O}$ is inhibited in $C$. annuum cv. Yolo $\mathrm{Y}$ as a result of homozygosity of the resistance allele $y^{\mathrm{a}}$ (Arroyo et al. 1996), whereas the resistance allele pvr3 in $C$. annuит cv. Avelar does not support long-distance movement of PepMoV (Murphy et al. 1998). The combination of two unlinked recessive resistance loci in tobacco N. tabacum cv. V20 also fails to support long-distance movement of TEVHAT (Schaad et al. 1997).

Recessive potyvirus resistance genes are often isolate or strain specific. Recent advances in constructing infectious cDNA clones of the positive-stranded potyvirus RNA genomes allowed for the generation of chimeras among potyvirus strains and the mapping of viral cistrons affected by different resistances. The genome-linked protein ( $\mathrm{VPg}$ ) encoded by the potyviruses has been identified as avirulence determinant of recessive resistances affecting replication (Keller et al. 
1998) and cell-to-cell (Nicolas et al. 1997) and long distance movement (Schaad et al. 1997). VPg of PSbMV determines virulence on $P$. sativum PI 269818 homozygous for the resistance gene sbm-1 (Keller et al. 1998). Here, we describe further analysis of the VPg coding region and the $s b m-1$ resistance mechanism.

\section{RESULTS}

\section{N terminal of PSbMV VPg protein determines virulence} in pea line PI 269818 homozygous for sbm-1.

By generating chimeras of PSbMV pathotype P1 isolate DPD1 and P4 isolate NY, the VPg cistron was shown to determine virulence on pea introduction line PI 269818 homozygous for the $s b m-1$ resistance gene (Keller et al. 1998). The VPg cistron was analyzed further by generating four additional virus chimeras between DPD1 and NY. Chimeras $\mathrm{NY}(\mathrm{DPD} 1-\mathrm{VPgN})$ and $\mathrm{NY}(\mathrm{DPD} 1-\mathrm{VPgC})$ replaced amino acids 1 to 122 from the $\mathrm{N}$ terminal or amino acids 123 to 194 from the $\mathrm{C}$ terminal of NY VPg with the corresponding DPD1 VPg sequences. In the reverse chimeras DPD1(NY-VPgN) and DPD1(NY-VPgC) with a DPD1 backbone, the N- and Cterminal VPg regions were substituted with NY VPg sequences. After the establishment of infection in pea cultivar Dark Skinned Perfection, each of the chimeric viruses were inoculated manually to 12 PI 269818 and 12 Dark Skinned Perfections as a control of inoculation efficiency. At 3 weeks postinoculation, PSbMV coat protein was detected in uninoculated leaves of all Dark Skinned Perfection plants inoculated with each of the four chimeras and in all of the PI 269818 inoculated with NY(DPD1-VPgC) or DPD1(NY-VPgN). None of the PI 269818 plants inoculated with NY(DPD1$\mathrm{VPgN})$ or DPD1(NY-VPgC) contained PSbMV coat protein at levels detectable by enzyme-linked immunosorbent assay (ELISA). Thus, the region encoding $122 \mathrm{~N}$-terminal amino acids of DPD1 VPg was sufficient to provide avirulence to isolate NY in the NY(DPD1-VPgN) chimera when inoculated to PI 269818. Correspondingly, the region encoding 122 amino acids of NY VPg in DPD1(NY-VPgN) chimera rendered DPD1 insensitive to the resistance. We therefore conclude that the N-terminal 122 amino acids of VPg determine NY/DPD1 virulence/avirulence in PI 269818.

\section{Mutational analysis of VPg.}

To identify amino acids affecting virulence/avirulence to PI 269818, we aligned the 122 amino acid N-terminal parts of DPD1 VPg and NY VPg (Fig. 1). The N-terminal parts of VPg protein from the two isolates were $82.8 \%$ identical. The most obvious block of differences were mapped in the region spanning positions 105 to 119 . Initially, sequences encoding amino acids 105 and 106 and 115 to 117 were selected for mutagenesis to test whether substitutions at two, three, or all five positions combined could provide virulence to DPD1 when inoculated to PI 269818 plants. Similarly, we replaced sequences encoding the two, three, and five amino acids in NY with codons corresponding to the amino acids from DPD1 to test whether any of these would affect NY infectivity on PI 269818. The DPD1 construct with two codon substitutions was made by changing methionine (Met) 105 to valine (Val) and tryptophan (Trp) 106 to phenylalanine (Phe). In the construct with three codon substitutions, Phe, Val, and threonine
(Thr), at positions 115 to 117 , were altered to Val, arginine (Arg), and serine (Ser). The two constructs were combined to generate a construct with five codon substitutions. The resulting mutant viruses were named DPD1-VF, DPD1-VRS, and DPD1-VF.VRS, respectively. The corresponding codon substitutions were introduced into the NY full-length clone, resulting in mutant viruses NY-MW, NY-FVT, and NYMW.FVT (Fig. 1).

Infection was established upon manual inoculation of Dark Skinned Perfection with plasmid DNA. Sap from systemically infected leaves was used to manually inoculate at least $12 \mathrm{PI}$ 269818 and 12 Dark Skinned Perfection controls for each of the six mutant viruses. At 3 weeks postinoculation, uninoculated leaves were tested by ELISA (Table 1). All but five out of 156 inoculated Dark Skinned Perfection plants were infected systemically, demonstrating the efficiency of the inoculation procedure and the infectivity of the mutant viruses. Mutant DPD1-VRS infected 23 out of 24 PI 269818, whereas DPD1-VF.VRS infected 20 out of 24 PI 269818. No infection was detectable by ELISA in uninoculated leaves of PI 269818 plants inoculated with DPD1-VF, NY-MW, NY-FVT, or NYMW.FVT. These results demonstrate that DPD1 became virulent on PI 269818, if the amino acids Phe-Val-Thr at positions 115 to 117 were substituted with Val-Arg-Ser, whereas NY was unable to infect PI 269818 if the amino acids Val-Phe at positions 105 and 106 were substituted with Met-Trp or ValArg-Ser at position 115 to 117 were substituted with Phe-ValThr.

\section{Analysis of resistance in inoculated leaves by $\beta$-glucuronidase tagged viruses.}

$\beta$-glucuronidase (GUS) tagged variants of the viruses (Fig. 1B) were expressed from constructs generated by inserting GUS between P1 proteinase (P1pro) and the helper component proteinase (HCpro), as described by Dolja et al. (1992). A superscript GUS was added to the virus names to indicate the presence of the GUS tag in the viruses. Arg was present at position 116 in the VPg of two other isolates that are infectious on PI 269818 (I. E. Johansen, unpublished observations). Therefore, we made additional GUS-tagged constructs of DPD1 and NY encoding a single amino acid substitution at position 116. In these two mutants, DPD $1^{\mathrm{GUS}}-\mathrm{R}$ and $\mathrm{NY}^{\mathrm{GUS}}-\mathrm{V}$. position 116 of VPg was changed from Val to Arg in DPD1 and from Arg to Val in NY (Fig. 1). A nonreplicating mutant, DPD1 ${ }^{\text {GUS }}$-VNN, encoding a defective RNA-dependent RNA polymerase was constructed as described for TEV (Carrington et al. 1993) by changing sequences encoding the conserved Gly351-Asp352-Asp353 motif to Val-Asn-Asn. DPD1 ${ }^{\mathrm{GUS}}$ VNN was used as a reference for GUS activity resulting from transient expression (Fig. 2A1 to A4). The GUS-tagged cDNA constructs were inoculated directly into PI 269818 and Dark Skinned Perfection plants by particle bombardment to avoid a virus amplification step, during which virus variants with deletions in the GUS reporter gene may accumulate (Dolja et al. 1993). In situ GUS assays of inoculated and noninoculated leaves were used to determine infection profiles (Table 1). PI 269818 plants were assayed repeatedly up to five weeks postinoculation to identify late-appearing, resistance-breaking variants.

After 2 weeks, all tagged viruses could be detected by GUS staining in inoculated (Fig. 2B3 to F3) and uninoculated 
leaves (data not shown) of every Dark Skinned Perfection plant inoculated by particle bombardment, demonstrating the efficiency of direct inoculation with plasmid DNA. At 1 week postinoculation, $\mathrm{NY}^{\mathrm{GUS}}$ (Fig. 2C1) and mutants DPD1 ${ }^{\mathrm{GUS}}$ VRS, DPD1 $1^{\text {GUS }}$-VF.VRS, and DPD ${ }^{\mathrm{GUS}}$-R (Fig. 2D1) were detected in all inoculated leaves of PI 269818 as infection foci several millimeters in diameter. In several of the plants, these mutants also were detected in uninoculated leaves. At 2 weeks postinoculation, all plants inoculated with $\mathrm{NY}^{\mathrm{GUS}}$ (Fig. 2C2), DPD1 ${ }^{\text {GUS }}$-VRS, DPD $1^{\text {GUS }}-$ VF.VRS, and DPD $1^{\text {GUS }}$-R (Fig. 2D2) cDNA constructs were infected systemically. DPD 1 GUS (Fig. 2B1 and B2) and mutants $\mathrm{NY}^{\mathrm{GUS}}$-MW, NY ${ }^{\mathrm{GUS}}$-FVT, $\mathrm{NY}^{\mathrm{GUS}}$-MW.FVT, and NYGUS-V (Fig. 2E1 and E2) were not detected by GUS assays in inoculated or uninoculated leaves of PI 269818 by 5 weeks postinoculation. To test whether the absence of GUS staining was a result of deletions in the GUS sequence rather than the absence of virus infection, RNA was isolated from inoculated plants and tested with reverse transcription-polymerase chain reaction (RT-PCR) with a primer set designed to amplify the VPg sequence. All plants that were negative in GUS assays were negative when tested by RTPCR, confirming that these plants were not infected. On PI 269818 leaves inoculated with DPD1 ${ }^{\mathrm{GUS}}$ (Fig. 2B1), NYGUS MW, NY GUS -FVT, NY ${ }^{\text {GUS }}$-MW.FVT, and NYGUS-V (Fig. 2E1), small blue inoculation foci were observed from 1 week postinoculation. These small foci were of the same size and intensity as foci observed upon bombardment with DPD1 ${ }^{\mathrm{GUS}}$ VNN (Fig. 2A1 and A3) and did not expand further during the experimental period.

Uninoculated leaves from PI 269818 plants inoculated with the mutant DPD1 ${ }^{\text {GUS }}$-VF were all negative in GUS assays 2 weeks postinoculation. In later tests at 4 and 5 weeks postinoculation, however, one of eight plants became systemically infected. Inoculation with DPD $1^{\text {GUS }}$-VF was repeated, and three of the 27 PI 269818 plants became systemically infected, displaying extensive blue staining in uninoculated leaves. On the inoculated leaves of these three plants, only a single expanding infection focus was detected in a background of many small nonexpanding inoculation foci (Fig. 2F2). On inoculated leaves of plants that remained negative throughout the experimental period, no such expanding foci were observed (Fig. 2F1). Infection of the three PI 269818 plants with mutant DPD1 ${ }^{\text {GUS }}-\mathrm{VF}$ was confirmed by RT-PCR. RT-PCR analysis of 10 of the 24 plants that did not show any sign of infection in GUS assays confirmed that these were not infected.

Except for these three PI 269818 plants inoculated with DPD1 ${ }^{\text {GUS }}$-VF, the inoculations by particle bombardment with GUS-tagged mutants were consistent with results obtained with mechanical inoculation followed by detection of the virus by ELISA. Tagging the virus mutants with GUS enabled us to analyze the extent of infection in inoculated leaves. Experiments had shown us that GUS activity in PI 269818 leaves inoculated with DPD1 ${ }^{\text {GUS }}$ (Fig. 2B1) did not exceed transient expression from a 35S-promoter-GUS construct (I. E. Johansen, unpublished observations) or the replicationdebilitated DPD1 $1^{\text {GUS }}$-VNN mutant in PI 269818 or Dark Skinned Perfection leaves (Fig. 2A1 and A3). Visual inspections of bombarded leaves provided no evidence that DPD1 ${ }^{\mathrm{GUS}}$ or mutants $\mathrm{NY}^{\mathrm{GUS}}$-MW, NY ${ }^{\mathrm{GUS}}$-FVT, NYGUS MW.FVT, and NYGUS-V did replicate in PI 269818 because
GUS activity did not exceed levels observed from transient expression. This suggests that the small blue foci seen in PI 269818 plants bombarded with DPD1 ${ }^{\text {GUS }}$ and mutants NYGUS MW, NY ${ }^{\mathrm{GUS}}$-FVT, NY ${ }^{\mathrm{GUS}}$-MW.FVT, NY ${ }^{\mathrm{GUS}}-\mathrm{V}$, and DPD1 ${ }^{\mathrm{GUS}}$ VF (except three plants) result only from transient GUS expression.

\section{Host-specific adaptation of VPg in PI 269818.}

In order to analyze the stability of the mutations in VPg of those mutants that overcame sbm-1 resistance in PI 269818, total RNA was recovered from uninoculated leaves of PI

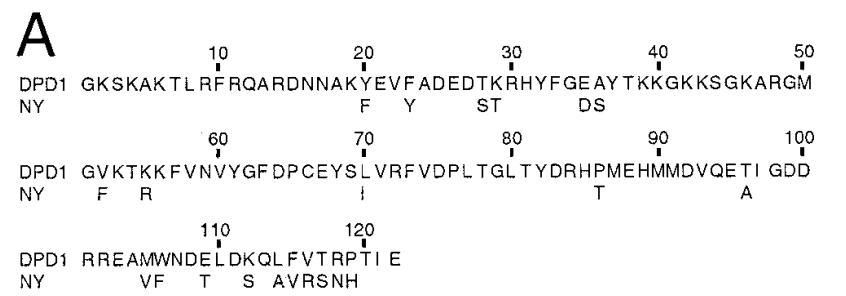

B

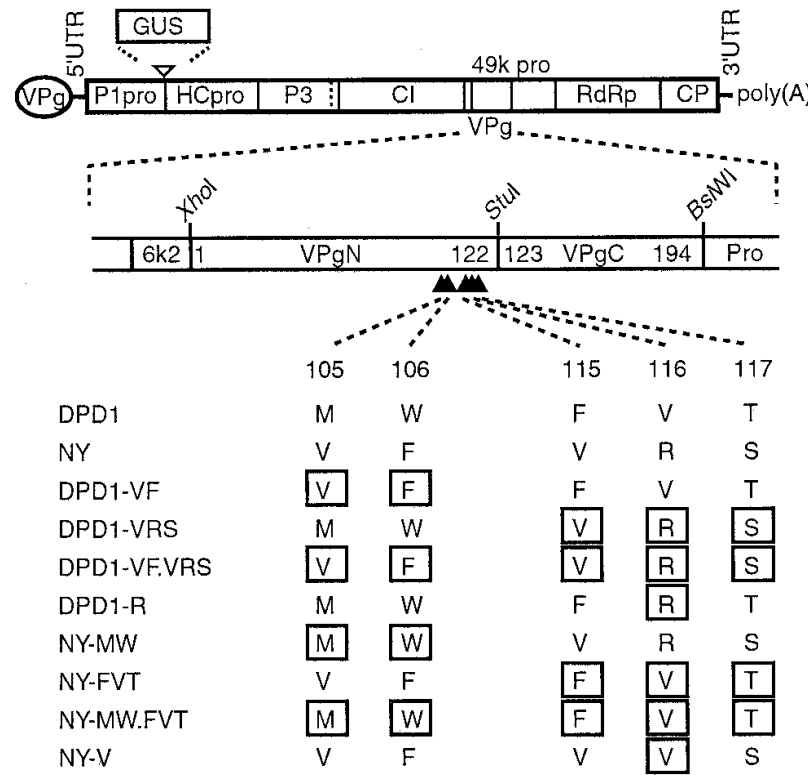

Fig. 1. Viral genome-linked protein (VPg) sequences and localization of mutations in DPD1 and NY. A, Alignment of the 122 amino acids Nterminal portion of VPg from DPD1 and NY. The NY sequence not shown is identical to DPD1. B, Cistron map of pea seedborne mosaic virus (PSbMV) indicating the position of the $\beta$-glucuronidase gene (GUS) insertion in tagged viruses. Enlargement of the VPg region shows restriction sites $X h o \mathrm{I}, S t u \mathrm{I}$, and $B s i \mathrm{WI}$, which were used to generate $\mathrm{VPgN}$ (codons 1-122) and VPgC (codons 123-194) chimeras of DPD1 and NY. Filled triangles below the VPgN fragment mark the positions of amino acids $105,106,115,116$, and 117 , which were mutated according to the scheme at the bottom of the figure. Unboxed amino acids are native to the virus, whereas boxed amino acids are mutated. Mutants were named after the native virus followed by the one-letter amino acid codes for the mutated amino acids. The untranslated regions (UTR) of PSbMV are indicated by horizontal lines, the coding region as an open box, and putative proteolytic cleavage sites as vertical lines. P1pro: P1 proteinase; HCpro: helper component proteinase; P3: third protein; 6k1: 6-kDa protein 1; CI: cylindrical inclusion protein-helicase; 6k2: 6-kDa protein 2; 49k pro: 49-kDa proteinase; VPg: genome-linked protein; RdRp: putative RNA-dependent RNA polymerase; CP: coat protein. 
269818 and Dark Skinned Perfection. The sequence of the VPg coding region corresponding to nucleotide (nt) 6,040 to 6,609 (amino acid 11 to 194 of VPg and amino acid 1 to 6 of the proteinase domain of NIa) was determined on RT-PCRamplified cDNA (Table 2). First, the mutant DPD1 ${ }^{\text {GUS }}$-VF was analyzed to make sure that the three infected PI 269818 plants were not a result of contamination. We found that the Val-Phe codons at position 105 and 106 were retained, but a lysine (Lys) codon at position 112 was changed to Thr in virus obtained from all three plants. In eight Dark Skinned Perfection plants, the DPD1 ${ }^{\mathrm{GUS}}$-VF mutant VPg sequence was retained unchanged. Analysis of DPD1 $1^{\text {GUS }}-$ VRS, DPD1 $1^{\text {GUS }}-$ VF.VRS, and DPD ${ }^{\text {GUS }}$-R showed that the mutant VPg sequences were retained unchanged in Dark Skinned Perfection. In PI 269818, the mutant VPg sequence of DPD1 ${ }^{\text {GUS }}$-VRS was unchanged in eight plants, the Met codon at position 105 was changed to leucine in three plants and isoleucine in one plant. The mutant VPg sequence of DPD1 ${ }^{\text {GUS }}$-VF.VRS was retained only in one PI269818 plant, whereas in four plants, the asparagine (Asn) codon at position 107 was changed to aspartic acid (Asp), and in three plants, the codon at position 112 was changed from Lys to Thr. Mutant VPg sequence of DPD1 ${ }^{\text {GUS }}$-R was retained in six plants. In one plant, position 107 changed from an Asn to an Asp codon, and in another plant, Phe codon 115 had changed to tyrosine. In all of the mutants, we observed no reversions in the codons that had been mutated. All the codon changes were in close vicinity to the introduced mutations and only affected position 105, 107, 112 and 115 (Table 2).

The changes observed in virus sequences that were amplified from PI 269818 could be the result of adaptation of the mutated viruses or of mutagenic properties of PI 269818 plants. In the latter case, we would expect this mutagenic property also to affect $\mathrm{NY}^{\mathrm{GUS}}$. Therefore, $\mathrm{VPg}$ sequences from eight PI 269818 plants inoculated with NYGUS were determined. No changes were observed in the sequenced area, suggesting that the changes seen with the VPg mutants were a result of adaptations of the virus to this host and not a mutagenic property of PI 269818.

Table 1. Infectivity of viral genome-linked protein mutant viruses detected by enzyme-linked immunosorbent assay (ELISA) and in situ $\beta$ glucuronidase (GUS) assays

\begin{tabular}{lcccc}
\hline & \multicolumn{2}{c}{$\begin{array}{c}\text { No. of systemically } \\
\text { infected }^{2} \text { plants }^{\mathbf{a}}\end{array}$} & \multicolumn{2}{c}{$\begin{array}{c}\text { No. of plants } \\
\text { expressing GUS }\end{array}$} \\
\cline { 2 - 5 } Virus & PI 269818 & DSP $^{\mathbf{c}}$ & PI 269818 & DSP \\
\hline DPD1 & $0 / 24$ & $22 / 24$ & $0 / 8$ & $4 / 4$ \\
NY & $22 / 22$ & $23 / 24$ & $8 / 8$ & $4 / 4$ \\
DPD1-VF & $0 / 24$ & $24 / 24$ & $3 / 27$ & $8 / 8$ \\
DPD1-VRS & $23 / 24$ & $23 / 24$ & $12 / 12$ & $4 / 4$ \\
DPD1-VF.VRS & $20 / 24$ & $24 / 24$ & $8 / 8$ & $4 / 4$ \\
NY-MW & $0 / 24$ & $12 / 12$ & $0 / 8$ & $4 / 4$ \\
NY-FVT & $0 / 12$ & $12 / 12$ & $0 / 8$ & $4 / 4$ \\
NY-MW.FVT & $0 / 12$ & $11 / 12$ & $0 / 8$ & $4 / 4$ \\
DPD1-R & nd & nd & $8 / 8$ & $4 / 4$ \\
NY-V & nd & nd & $0 / 8$ & $4 / 4$ \\
\hline
\end{tabular}

${ }^{a}$ Number of ELISA-positive plants/number of plants tested. Plants were inoculated with sap from plants infected with untagged viruses.

${ }^{\mathrm{b}}$ Number of plants with GUS staining/number of plants tested. Plants were inoculated by particle bombartment with GUS-tagged cDNA constructs. All positive plants showed GUS staining in inoculated and uninoculated leaves.

${ }^{c}$ Pisum sativum L cv. Dark Skinned Perfection.

${ }^{\mathrm{d}}$ Not determined.
Native DPD1 and NY VPg and all mutated VPg proteins were analyzed by tools that predict primary structure from the ExPaSy Server, which were provided by the Swiss Institute of Bioinformatics (Appel et al. 1994). We found no structural predictions that consistently suggest a difference between constructs that were sensitive and insensitive to $s b m-1$ resistance.

\section{DPD1 VPg did not induce resistance in PI $269818 \mathrm{sbm}-1 / \mathrm{sbm}-1$ plants.}

The resistance to DPD1 in PI 269828 plants could be the result of a defense response elicited upon expression of DPD1 VPg. This was tested initially in coinoculation experiments with preinoculation of DPD1, followed by NY inoculation 4 days latr, and mixed inoculum of DPD1 and NY. These experiments did not provide any evidence that DPD1 induced a defense mechanism in PI269818 that affected NY infectivity on these plants. If the defense reaction does not spread beyond the inoculated cell, however, the defense response will affect both viruses only if they are present in the same cell. It could be argued that in coinoculation experiments, there is no guarantee that both viruses enter the same cells. To circumvent this problem, a NY clone was constructed that also carried the 6k2-VPg-Pro coding region from DPD1 inserted between P1pro and HCpro. This large segment was chosen to put DPD1 VPg in a native context that could be processed by the homologous proteinase domain. The construct, $\mathrm{NY}^{+\mathrm{DPD} 16 \mathrm{k2}-\mathrm{VPg}-}$ Pro, was inoculated by particle bombardment to Dark Skinned Perfection and PI 269818 and tested by ELISA 3 weeks postinoculation. All plants of Dark Skinned Perfection and PI 269818 were infected systemically. A series of RT-PCR controls were performed to determine whether the viruses might have lost the inserted DPD1 region or the inserted region had recombined with homologous sequences of the NY backbone. RNA from five Dark Skinned Perfection plants all produced RT-PCR amplification fragments consistent with a NY virus carrying an intact DPD1 6k2-VPg-pro region as did nine of 14 PI 269818 plants. RNA from the remaining five PI 269818 plants did not yield the expected PCR fragments or gave rise to a fragment consistent with a deletion in the DPD1 6k2VPg-Pro region (data not shown). Thus, in at least nine PI 269818 plants, the DPD1 6k2-VPg-Pro region was intact. This experiment did not support a hypothesis that DPD1 VPg elicits a defense response to PSbMV in PI 269818.

\section{Co-expression of NY VPg did not complement DPD1 VPg function.}

Previously it was investigated whether NY was able to complement DPD1 in PI 269818 by coinoculation experiments. Keller at al. (1998) failed to detect any complementation either by preinoculation of NY followed by inoculation of DPD1 or simultaneous inoculation with NY and DPD1. Again, it can be argued that it is not certain whether both viruses enter the same cells. Therefore we constructed a DPD1 cDNA clone that also carried the 6k2-VPg-Pro coding region of NY inserted between P1pro and HCpro to be sure that NY VPg was expressed in the same cells as DPD1. We chose to insert this large segment in order to put NY VPg in a native context that could be processed by the homologous proteinase domain. The construct, DPD ${ }^{+\mathrm{NY}}$ 6k2-VPg-Pro, was inoculated manually to Dark Skinned Perfection, and infected plant sap 
was used to inoculate eight Dark Skinned Perfection and 12 PI 269818 plants. These plants were tested by ELISA 3 weeks postinoculation, and all eight Dark Skinned Perfection plants were infected systemically, whereas none of the PI 269818 plants contained detectable PSbMV coat protein. The experiment was repeated by employing direct inoculation of the construct by particle bombardment to Dark Skinned Perfection and PI 269818. At 3 weeks postinoculation, all Dark Skinned Perfection plants were infected systemically, whereas two of the 10 PI 269818 plants were positive in ELISA. Both of these plants were tested by RT-PCR but failed to produce the expected fragments. Thus, our results are in agreement with Keller et al. (1998) because we did not find that NY VPg could complement DPD1 in PI 269818, suggesting that the VPg function that is affected by sbm-1 resistance depends upon expression from the native position in the genome.

\section{DISCUSSION}

We presented data demonstrating that only minor changes in the central region of VPg spanning positions 105 to 117 were required to alter virulence of PSbMV isolates DPD1 and NY on PI line 269818 homozygous for the resistance gene sbm-1. When Val 116 in avirulent DPD1 was substituted with Arg, the mutant virus systemically infected PI 269818. Infectivity in PI 269818, however, depended upon Arg 116 as well as the VPg context because NY-MW was avirulent on PI 269818, although this mutant still carried Arg 116. We also observed that DPD1-VF, which still had Val 116, was virulent on a few PI 269818 plants. Infection by this virus was significantly delayed, and virus recovered from these plants all contained a Lys to Thr substitution at position 112. This change was not seen in Dark Skinned Perfection plants, suggesting that this adaptation was necessary for infection of PI 269818.

In a previous study, transfection of PI 269818 protoplasts did not result in the measurable replication of DPD1, suggesting that the resistance operates at the replication level (Keller et al. 1998). An efficient block of an early step in replication could explain why we never observed spontaneous resistance- breaking mutants such as DPD1-R in PI 269818. Mutant progeny will not arise during RNA synthesis if replication is completely blocked in resistant plants. If just a few rounds of replication are possible, however, it could be expected that the virus, at least in a few cases, generates spontaneous mutations that break resistance. Such a scenario could explain our observations on the mutant DPD1 ${ }^{\mathrm{GUS}}$-VF that produced a delayed infection only in a few PI 269818 test plants. Recovered progeny virus all contained an additional Lys to Thr mutation at position 112. This suggests that mutant VPg of DPD1-VF and DPD1 ${ }^{\text {GUS }}$-VF did not function efficiently in PI 269818 and only upon efficient inoculation by particle bombardment was infection occasionally established. Infections may arise if a few inefficient rounds of replication allow for the generation of spontaneous mutations that overcome resistance. This would also be consistent with our observation that only one infection focus was observed on inoculated leaves of PI 269818 that did become infected by DPD1 ${ }^{\text {GUS }}$-VF (Fig. 2F2).

The DPD1 ${ }^{+N Y}$ 6k2-VPg-Pro construct was inoculated to test whether NY VPg could complement DPD1 in PI 269818 when expressed as an extra protein. We did not detect any infection of PI 269818, although the construct was fully infectious on Dark Skinned Perfection, indicating that VPg must be expressed from its native position in the genome to overcome resistance. This agrees with the observation by Schaad et al. (1996) that VPg-Pro functions of TEV cannot be complemented by transgenic expression, suggesting that VPg-Pro provides replicative functions that are cis preferential.

Resistance affecting cell-to-cell and long-distance movement also depended on VPg (Hämäläinen et al. 2000; Nicolas et al. 1997; Rajamäki and Valkonen 1999; Schaad et al. 1997). The va resistance gene from $N$. tabacum cv. VAM operates mainly at the level of cell-to-cell movement (Gibb et al. 1989). Four amino acid changes in the center of VPg of TVMV-WT were needed to efficiently break resistance (Nicolas et al. 1997). The positions of these four amino acids correspond to positions 110, 111, 114, and 115 of PSbMV VPg. In PVA VPg, the amino acid corresponding to position 114 of PSbMV VPg is the major determinant of systemic in-

Table 2. Analysis of viral genome-linked protein (VPg) sequence recovered from infected plants

\begin{tabular}{|c|c|c|c|c|}
\hline Virus & Pea line & No. of plants expressing $\beta$-glucuronidase (GUS) ${ }^{\mathbf{a}}$ & Recovered VPg sequence $^{\mathrm{b}}$ & No. of plants ${ }^{c}$ \\
\hline \multirow[t]{2}{*}{ DPD1 $1^{\text {GUS }}-\mathrm{VF}$} & $\mathrm{DSP}^{\mathrm{d}}$ & 8 & VFNDELDKQLFVT & 8 \\
\hline & PI 269818 & 3 & VFNDELD辔QLFVT & 3 \\
\hline \multirow[t]{4}{*}{ DPD1 ${ }^{\text {GUS }}-$ VRS } & DSP & 4 & MWNDELDKQLVRS & 4 \\
\hline & PI 269818 & 12 & MWNDELDKQLVRS & 8 \\
\hline & & & $\underline{\mathbf{L} W N D E L D K Q L V R \mathbf{S}}$ & 3 \\
\hline & & & $\underline{\mathbf{I}} W N D E L D K Q L \mathbf{V R} \mathbf{S}$ & 1 \\
\hline \multirow[t]{4}{*}{ DPD1 ${ }^{\text {GUS }}$-VF.VRS } & DSP & 4 & VFNDELDKQLVRS & 4 \\
\hline & PI 269818 & 8 & VFNDELDKQLVRS & 1 \\
\hline & & & 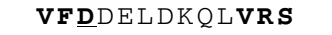 & 4 \\
\hline & & & VF NDELDTQLVRS & 3 \\
\hline \multirow[t]{4}{*}{$\mathrm{DPD} 1^{\mathrm{GUS}}-\mathrm{R}$} & DSP & 4 & MWNDELDKQLF RT & 4 \\
\hline & PI 269818 & 8 & MWNDE L DKQ L F RT & 6 \\
\hline & & & $\underline{\mathbf{I}} W N D E L D K Q L F \mathbf{R T}$ & 1 \\
\hline & & & MWNDELDKQ L $\underline{\mathbf{Y} R T}$ & 1 \\
\hline \multirow{2}{*}{ NY ${ }^{\text {GUS }}$} & DSP & 4 & VFNDTLDSQAVRS & 4 \\
\hline & PI 269818 & 8 & VFNDTLDSQAVRS & 8 \\
\hline
\end{tabular}


fection in Nicandra physaloides (Rajamäki and Valkonen 1999). It is intriguing that recessive resistance genes sbm-1 and $v a$ and resistance in $N$. physaloides depend upon the same region in the central VPg because three different steps in infection apparently are affected. Whether this means that the central region of VPg interacts with several host factors supporting replication and cell-to-cell and long-distance movement or whether the same host factor contributes to several of the mentioned viral requirements remains to be investigated. It also is possible that VPg serves distinct functions in different virus-host combinations or that it induces a host defense reaction that is activated differently in different host plants. Coinoculation experiments have not provided evidence that VPg can induce a defense reaction (Keller et al. 1998; Nicolas et al. 1997; Rajamäki and Valkonen 1999; Schaad et al. 1997). In these types of experiments, it is expected that a defense reaction induced by the avirulent strain also will affect the coinoculated virus. It cannot be ruled out that a defense response may be active only at the single-cell level, and coinoculation failed to deliver both viruses to the same cells. To test this possibility, we constructed a NY variant carrying the 6k2VPg-Pro coding region from DPD1 inserted between the P1pro and HCpro cistrons. We found that this construct, $\mathrm{NY}^{+\mathrm{DPD} 16 \mathrm{6} 2 \text {-VPg-Pro }}$, was fully infectious on PI 269818. Thus, we were not able to provide any evidence for a defense reaction that was active only at the single-cell level.

It previously has been hypothesized that the dominant alleles of genes conferring recessive resistance encode host factors required for virus infection. The eukaryotic translation initiation factors eIF(iso)4E and eIF4E are as yet the only host proteins that interact with potyviral VPg (Schaad et al. 2000; Wittmann et al. 1997). The interaction domain was mapped to a stretch of 35 amino acids of turnip mosaic virus VPg and, within this domain, the mutation of Asp 77 to Asn abolished in vitro interaction and viral infectivity (Léonard et al. 2000). Asp 77 corresponds to Asp 75 in PSbMV, which is conserved
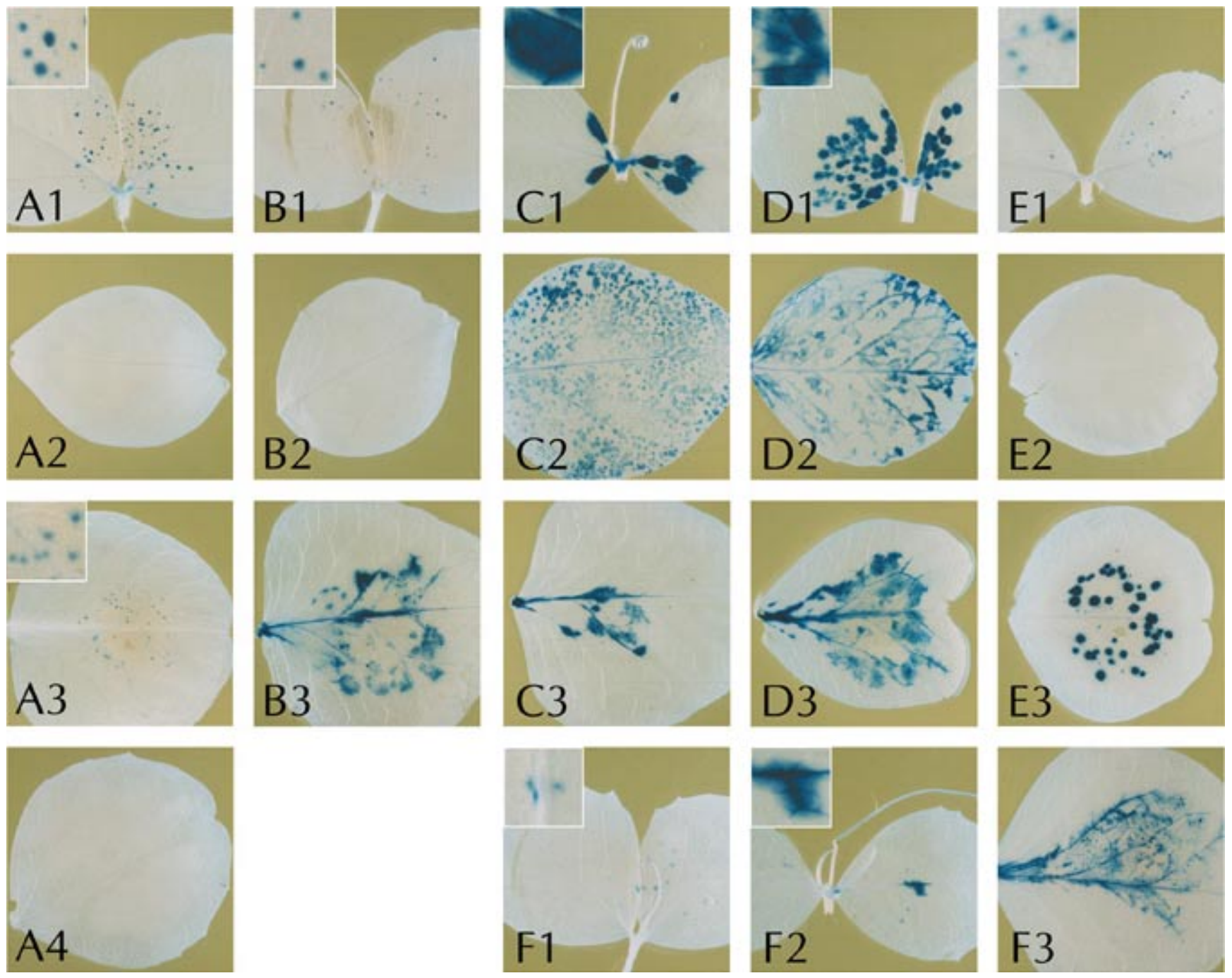

Fig. 2. In situ $\beta$-glucuronidase (GUS) assays of pea leaves from plants inoculated with GUS-tagged pea seedborne mosaic virus (PSbMV) constructs A1-4, DPD1 ${ }^{\mathrm{GUS}}-\mathrm{VNN}, \mathbf{B 1 - 3}$, DPD1 ${ }^{\mathrm{GUS}}, \mathbf{C 1 - 3}, \mathrm{NY}^{\mathrm{GUS}}, \mathrm{D1}-\mathbf{3}$, DPD1 ${ }^{\mathrm{GUS}}-\mathrm{R}, \mathbf{E 1 - 3}, \mathrm{NY}^{\mathrm{GUS}}-\mathrm{V}$, and F1-3, DPD1 ${ }^{\mathrm{GUS}}-\mathrm{VF}$. A1-E1, Inoculated leaves of PI 26981812 days postinoculation (dpi). A2-E2, Uninoculated leaves of PI 269818 at 12 dpi. A3-E3, Inoculated leaves of Pisum sativum L cv. Dark Skinned Perfection at 12 dpi. A4, Uninoculated leaf of Dark Skinned Perfection inoculated with DPD1 ${ }^{\text {GUS }}$-VNN. F1 and F2, DPD1 ${ }^{\text {GUS }}$-VF-inoculated leaves of PI $26981821 \mathrm{dpi}$. F3, Inoculated Dark Skinned Perfection leaf at $12 \mathrm{dpi}$. Insets represent enlargements of $1.75 \mathrm{~mm}^{2}$. 
in all PSbMV isolates. It is therefore unlikely that the gene product of $S b m-1$ is a pea version of eIF(iso)4E or eIF4E.

\section{MATERIALS AND METHODS}

Virus isolates and plants.

The PSbMV isolates DPD1 and NY representing pathotypes P1 and P4 have been described previously (Johansen et al. 1991; Johansen et al. 1996) and are available as infectious cDNA clones (Johansen 1996). Infectious recombinant clones P1(P4 VPg) and P4(P1 VPg) were described by Keller et al. (1998). Seeds of Pisum sativum L cv. Dark Skinned Perfection were obtained from Dænfeldt (Denmark), and plant introduction line (PI) 269818 was provided by K. Keller, USDA-ARS (Corvallis, OR, U.S.A.).

\section{cDNA cloning, mutagenesis, and sequencing.}

All cloning experiments were performed with standard protocols (Sambrook et al.1989). Modifying enzymes were purchased from Roche Diagnostics (Mannheim, Germany), unless stated otherwise. Nucleotide changes in the viral cDNA sequences were engineered by PCR with $P f u$ polymerase (Stratagene, La Jolla, CA, U.S.A.) and the appropriate oligonucleotide primers from DNA Technology (Aarhus, Denmark). DNA sequencing reactions were analyzed on an automated Perkin Elmer ABI 377XL DNA sequencer (Norwalk, CT, U.S.A.).

To divide the VPg sequence into two parts, VPgN and $\mathrm{VPgC}$, a StuI site was introduced in the DPD1 coding sequence at nt 6,373 to 6,378 and the NY coding sequence at nt 6,311 to 6,316 (Fig. 1). Recombinant full-length clones DPD1(NY VPgN) and NY(DPD1 VPgN) were constructed by inserting the $S t u \mathrm{I}-B s i \mathrm{WI} \mathrm{VPgC}$ coding region of DPD1 and $\mathrm{NY}$ into clones $\mathrm{P} 1(\mathrm{P} 4 \mathrm{VPg})$ and $\mathrm{P} 4(\mathrm{P} 1 \mathrm{VPg})$, respectively. Clones DPD1(NY VPgC) and NY(DPD1 VPgC) were constructed by inserting the XhoI-StuI VPgN coding region of DPD1 and NY into P4(P1 VPg) and P1(P4 VPg).

A series of DPD1 and NY cDNA clones were generated with point mutations, altering the codons of amino acids in VPg at positions 105, 106, 115, 116, and 117 (Fig. 1). Sitedirected mutagenesis was performed on DPD1 and NY subclones containing the VPg coding sequence as a XhoI-BsiWI fragment. Mutations were verified by sequencing and mutated DPD1, and NY fragments were transferred to clones P1(P4 $\mathrm{VPg})$ and $\mathrm{P} 4(\mathrm{P} 1 \mathrm{VPg})$ by exchanging the $\mathrm{XhoI}-$ BsiWI region. Clones DPD $1^{\mathrm{GUS}}$ and $\mathrm{NY}^{\mathrm{GUS}}$ were tagged by the GUS gene, resulting in expression of a GUS-HCpro fusion, as described by Dolja et al. (1992). The GUS gene was excised from p306GUS as a NcoI fragment and inserted between P1pro and HCpro coding regions at a NcoI site engineered into the DPD1 sequence at nt 1,336 to 1,341 of DPD1 and nt 1,295 to 1,300 of NY. GUS-tagged variants of all mutant clones were generated by substituting the native VPg coding region with the corresponding region from individual mutant clones. Clone DPD $1^{+N Y} 6 \mathrm{k} 2-\mathrm{VPg}$-Pro was constructed by substituting GUS in DPD1 ${ }^{\text {GUS }}$ with the $6 \mathrm{k} 2-\mathrm{VPg}$-Pro coding region of NY. For this purpose, RcaI sites were introduced $5^{\prime}$ (nt 5,782 to 5,787) and $3^{\prime}$ (nt 7,271 to 7,276) to the 6k2-VPg-Pro coding region of NY. Clone NY+DPD1 6k2-vPg-Pro was constructed by substituting the GUS in NYGUS with the 6k2-VPg-Pro coding region of DPD1. In this case, a RcaI site was introduced $5^{\prime}$ (nt 5,845 to 5,850 ) and a coding region $N c o$ I site introduced $3^{\prime}$ (nt 7,330 to
7,335) to the 6k2-VPg-Pro coding region of DPD1. In both constructs (DPD1 ${ }^{+\mathrm{NY}} 6 \mathrm{k} 2$-VPg-Pro and $\mathrm{NY}^{+\mathrm{DPD} 16 \mathrm{k} 2 \text {-VPg-Pro }}$ ), the $3^{\prime}$ restriction site was placed after the predicted proteolytic cleavage site to allow cleavage between 6k2-VPg-Pro and HCpro.

\section{Virus inoculation and detection.}

Infection from PSbMV cDNA constructs without a GUS gene insertion was established by manual inoculation of Dark Skinned Perfection with the aid of carborundum. Sap from infected plants was subsequently used to inoculate test plants. Twelve test plants and 12 susceptible Dark Skinned Perfection plants were inoculated in each experiment as a control of inoculation efficiency. PSbMV constructs tagged with the GUS reporter gene were inoculated directly to test plants with the Helios Gene Gun System (BioRad Laboratories, Hercules, CA, U.S.A.). Two to four leaves of at least eight test plants and four susceptible control plants were inoculated with each construct.

Infectivity of untagged cDNA clones and viruses was determined 3 weeks after inoculation by assaying young leaves of inoculated plants for the presence of PSbMV coat protein by ELISA with a PSbMV-specific polyclonal antiserum (Kohnen et al. 1995). Plants were defined as infected if the ELISA value was at least four times higher than the mock control. Inoculated and uninoculated leaves of plants inoculated with GUS-tagged cDNA constructs were assayed at several time points after inoculation by in situ GUS assays (Dolja et al. 1992).

For PCR analysis, total RNA was extracted from $100 \mathrm{mg}$ of pea leaf tissue (Spiegel and Martin 1993) and reverse transcribed by M-MLV reverse transcriptase (GIBCO-BRL, Roskilde, Denmark) with an oligo(dT) primer. The VPg coding region, which had been subjected to mutagenesis, was amplified by PCR with primer pairs DPD1.VPg.fw (nt 6,040 to 6,062)-DPD1.VPg.rv1 (nt 6,609 to 6,586) and NY.VPg.fw (nt 6,029 to 6,049)- NY.VPg.rv1 (nt 6,529 to 6,511). PCR products were subjected to gel electrophoresis and purified with a gel extraction kit (Qiagen, Hilden, Germany) prior to sequencing. The PCR primers also were used for sequencing. Primer pairs DPD1.P1.fw (nt 1,031 to 1,061)-NY.VPg.rv2 (nt 6,301 to 6,285), DPD1.CI.fw (nt 5,464 to 5,480)DPD1.VPg.rv2 (nt 6,367-6,350), DPD1.P1.fwDPD1.VPg.rv2, and DPD1.CI.fw-NY.VPg.rv2 were used to verify the $\mathrm{DPD}^{+\mathrm{NY}}$ 6k2-VPg-Pro construct, and primer pairs NY.P1.fw (nt 702 to 727)-DPD1.VPg.rv2, NY.CI.fw (nt 5,673 to 5,694)-NY.VPg.rv2, NY.P1.fw-NY.VPg.rv2, and NY.CI.fwDPD1.VPg.rv2 to verify the $\mathrm{NY}^{+\mathrm{DPD} 16 \mathrm{k} 2 \text {-VPg-Pro }}$ construct.

\section{Sequence analysis.}

Physicochemical parameters and Chou and Fasman predictions of $\alpha$-helices, $\beta$-sheets, and $\beta$-turns of DPD1, NY, and all mutant VPg proteins were analyzed by the ProtParam and ProtScale tools from the ExPaSy Server provided by the Swiss Institute of Bioinformatics (Appel et al. 1994). VPg amino acid sequences were aligned by use of the CLUSTAL method provided by MegAlign software, which is part of the DNASTAR package (DNASTAR, Madison, WI, U.S.A.).

\section{ACKNOWLEDGMENTS}

We thank M. Albrechtsen and J. Laursen for fruitful discussions and critically reading the manuscript and $\mathrm{B}$. S Olsen for expert technical 
assistance. p306GUS was kindly provided by J. Carrington. Financial support was provided by grant BIO4-CT97-2356 from the biotechnology program of the European Union.

\section{LITERATURE CITED}

Appel, R. D., Bairoch, A., and Hochstrasser, D. F. 1994. A new generation of information retrieval tools for biologists: The example of the ExPaSy WWW server. Trends Biochem. Sci. 19:258-260.

Arroyo, R., Soto, M. J., Martínez-Zapater, J. M., and Ponz, F. 1996. Impaired cell-to-cell movement of potato virus $\mathrm{Y}$ in pepper plants carrying the $y^{\mathrm{a}}\left(p r 2^{1}\right)$ resistance gene. Mol. Plant-Microbe Interact. 9:314-318.

Carrington, J. C., Haldeman, R., Dolja, V. V., Restrepo-Hartwig, M. A. 1993. Internal cleavage and trans-proteolytic activities of the VPgProteinase (NIa) of tobacco etch potyvirus in vivo. J. Virol. 67:69957000.

Deom, C. M., Murphy, J. F., and Paguio, O. R. 1997. Resistance to tobacco etch virus in Capsicum annuum: Inhibition of virus RNA accumulation. Mol. Plant-Microbe Interact. 10:917-921.

Dolja, V. V., McBride, H. J., and Carrington, J. C. 1992. Tagging of plant potyvirus replication and movement by insertion of $\beta$-glucuronidase into the viral polyprotein. Proc. Natl. Acad. Sci. USA 89:1020810212.

Dolja, V. V., Herndon, K. L., Pirone, T. P., and Carrington, J. C. 1993. Spontaneous mutagenesis of a plant potyvirus genome after insertion of a foreign gene. J. Virol. 67:5968-5975.

Fraser, R. S. S. 1992. The genetics of plant-virus interactions: Implications for plant breeding. Euphytica 63:175-185

Freialdenhoven, A., Peterhänsel, C., Kurth, J., Kreuzaler, F., and Schulze-Lefert, P. 1996. Identification of genes required for the function of non-race-specific mlo resistance to powdery mildew in barley. Plant Cell 8:5-14.

Gibb, K. S., Hellmann, G. M., and Pirone, T. P. 1989. Nature of resistance of a tobacco cultivar to tobacco vein mottling virus. Mol. PlantMicrobe Interact. 2:332-339.

Hämäläinen, J. H., Kekarainen, T., Gebhardt, C., Watanabe, K. N., and Valkonen, J. P. T. 2000. Recessive and dominant genes interfere with the vascular transport of Potato virus $A$ in diploid potatoes. Mol. Plant-Microbe Interact. 13:402-412.

Johansen, E., Rasmussen, O. F., Heide, M., and Borkhardt, B. 1991. The complete nucleotide sequence of pea seed-borne mosaic virus RNA. J. Gen. Virol. 72:2625-2632.

Johansen, I. E. 1996. Intron insertion facilitates amplification of cloned virus cDNA in Escherichia coli while biological activity is reestablished after transcription in vivo. Proc. Natl. Acad. Sci. USA 93:12400-12405.

Johansen, I. E., Keller, K. E., Dougherty, W. G., and Hampton, R. O. 1996. Biological and molecular properties of a pathotype P-1 and a pathotype P-4 isolate of pea seed-borne mosaic virus. J. Gen. Virol. 77:1329-1333.

Keller, K. E., Johansen, I. E., Martin, R. R., and Hampton, R. O. 1998. Potyvirus genome-linked protein (VPg) determines pea seed-borne mosaic virus pathotype-specific virulence in Pisum sativum. Mol. Plant-Microbe Interact. 11:124-130.

Kohnen, P. D., Johansen, I. E., and Hampton, R. O. 1995. Characterization and molecular detection of the P4 pathotype of pea seedborne mosaic potyvirus. Phytopathology 85:789-793.

Léonard, S., Plante, D., Wittmann, S., Daigneault, N., Fortin, M. G., and Laliberté, J.-F. 2000. Complex formation between potyvirus VPg and translation eukaryotic initiation factor $4 \mathrm{E}$ correlates with virus infectivity. J. Virol. 74:7730-7737.

Masuta, C., Nishimura, M., Morishita, H., and Hataya, T. 1999. A single amino acid change in viral genome-associated protein of potato virus Y correlates with resistance breaking in 'Virgin A Mutant' tobacco. Phytopathology 89:118-123.

Murphy, J. F., Blauth, J. R., Livingstone, K. D., Lackney, V. K., and Jahn, M. K. 1998. Genetic mapping of the pvrl locus in Capsicum spp. and evidence that distinct potyvirus resistance loci control responses that differ at the whole plant and cellular levels. Mol. PlantMicrobe Interact. 11:943-951.

Nicolas, O., Dunnington, S. W., Gotow, L. F., Pirone, T. P., and Hellmann, G. M. 1997. Variations in the VPg protein allow a potyvirus to overcome va gene resistance in tobacco. Virology 237:452-459.

Noguchi, S., Tajima, T., Yamamoto, Y., Ohno, T., and Kubo, T. 1999. Deletion of a large genomic segment in tobacco varieties that are resistant to potato virus Y (PVY). Mol. Gen. Genet. 262:822-829.

Rajamäki, M.-L., and Valkonen, J. P. T. 1999. The 6K2 protein and the $\mathrm{VPg}$ of potato virus A are determinants of systemic infection in $\mathrm{Ni}$ candra physaloides. Mol. Plant-Microbe Interact. 12:1074-1081.

Revers, F., Le Gall, O., Candresse, T., and Maule, A. J. 1999. New advances in understanding the molecular biology of plant/potyvirus interactions. Mol. Plant-Microbe Interact. 12:367-376.

Riechmann, J. L., Laín, S., and García, J. A. 1992. Highlights and prospects of potyvirus molecular biology. J. Gen. Virol. 73:1-16.

Sambrook, J., Fritsch, E. F., and Maniatis, T. A. 1989. Molecular Cloning: A Laboratory Manual, 2nd ed. Cold Spring Harbor Laboratory, Cold Spring Harbor, NY, U.S.A.

Schaad, M. C., Haldeman-Cahill, R., Cronin, S., and Carrington, J. C. 1996. Analysis of the VPg-proteinase (NIa) encoded by tobacco etch potyvirus: Effects of mutations on subcellular transport, proteolytic processing, and genome amplification. J. Virol. 70:7039-7048.

Schaad, M. C., Lellis, A. D., and Carrington, J. C. 1997. VPg of tobacco etch virus is a host genotype-specific determinant for long-distance movement. J. Virol. 71:8624-8631.

Schaad, M. C., Anderberg, R. J., and Carrington, J. C. 2000. Strainspecific interaction of the tobacco etch virus NIa protein with the translation initiation factor eIF4E in the yeast two hybrid system. Virology 273:300-306.

Spiegel, S., and Martin, R. R. 1993. Improved detection of potato leafroll virus in dormant potato tubers and microtubers by the polymerase chain reaction and ELISA. Ann. Appl. Biol. 122:493-500.

Wittmann, S., Chatel, H., Fortin, M. G., and Laliberté, J.-F. 1997. Interaction of the viral protein genome linked of turnip mosaic potyvirus with the translational eukaryotic initiation factor (iso) 4E of Arabidopsis thaliana using the yeast two-hybrid system. Virology 234:8492. 